\title{
The Influence of Multispectral Radiation Thermometry by Mie Scattering
}

\author{
Chengda Ning ${ }^{a}$, Xianyong Jing ${ }^{b}$, Yuanyuan Sun ${ }^{c}$ and Zhihuan Lan ${ }^{d}$ \\ Air Force Aviation University, Changchun 130022, China

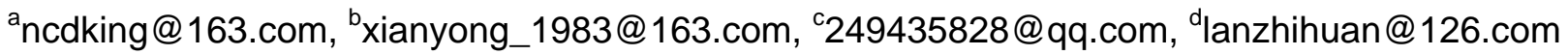

Keywords: Multi-spectral radiation thermometry, Mie scattering, Blackbody radiation, Scattering coefficient.

\begin{abstract}
In the research of multi-spectral radiation thermometry, temperature measurement depends on the line shape of spectrum radiation remittance. However, in the actual measurement, the combustion, explosion and gasification on special occasions can produce some particulate matters which can create the phenomena of scatterings such as Mie scattering, Rayleigh scattering and Debyd scattering. These phenomena will affect the linear of spectral line directly. The particulate matters can also make the object's line shape of spectrum radiation remittance changed through the transmission of scattering medium. Therefore, it will affect the precision of temperature measurement. Based on the theory of blackbody radiation, this thesis researches on not only the influence and revision of multi-spectral radiation thermometry by Mie scattering, but also the influence and revision of solar temperature measurement by the phenomenon of PM2.5 scattering. The thermal error will drop from $20 \%$ to $10 \%$ through the revision. The research will improve the precision of temperature measurement.
\end{abstract}

\section{Introduction}

Multi-spectral radiation thermometry is a new kind of non-contact temperature measuring method [1], the theory basis is the formula of black-body radiation, according to the spectrum radiation line type of the measured object, the black-body radiation line type is fitted, if black-body radiation line type is similar in the same temperature, then what is the temperature value.

The advantage of multi-spectral radiation thermometry is convenient which can measure high temperature object and high precision, enables this way to meet the difficulties faced by many industries in terms of temperature measurement [2]. But this way is totally dependent on spectrum radiation line type of object under test, if line type is interfered by outside factors and it will affect the accuracy of measurement, scattering characteristics of atmospheric spherical particles themselves have biggest effect in these influence factors, thus cause spectral line type changes in the process of transmission.

\section{Theoretical basis}

\subsection{Mie scattering basic theory}

Scattering is that the atmospheric gas molecules and small particles and other substances in the light, under the waves of oscillation, polarization phenomenon occurs, the interaction between polarization phenomenon and light waves produces electromagnetic multipole, these electromagnetic multipole radiate light wave in the form of light wave, this is scattering phenomenon.

When incident light of unit discharge irradiates the particles, the total energy of light scattered in all directions, and particle cross-sectional area ratio that is called the $K_{\text {sca }}$ scattering coefficient, total light energy absorbed by particles and the ratio of cross-sectional area is called the $K_{a b s}$ absorption coefficient, and the sum of $K_{s c a}$ and $K_{a b s}$ is called $K_{\text {ext }}$ extinction coefficient [4]. The specific expression is as follows:

$$
K s c a=\frac{2}{x^{2}} \sum_{n-1}^{m}(2 n+1)\left(\left|a_{n}\right|^{2}+\left|b_{n}\right|^{2}\right)
$$




$$
K_{\text {ext }}=\frac{2}{x^{2}} \sum_{n-1}^{m}(2 n+1) \operatorname{Re}\left(a_{n}+b_{n}\right)
$$

It has been seen from the formula, $K_{\text {sca }}$ is associated with $a_{n}, b_{n}$, and, $a_{n}, b_{n}$ are the determined by scattering coefficient $\mathrm{m}, \mathrm{n}, m$ are the complex refractive index of particles, $n$ is positive integer, $m_{r}$ and $m_{i}$ are respectively is real and imaginary part of refractive index, usually take $<0$ in the practical calculation, $\mathrm{a}_{\mathrm{n}}, \mathrm{b}^{\mathrm{n}}$ expression as follows [5].

$$
\begin{aligned}
& a_{n}=\frac{\psi_{n}(m \alpha) \psi_{n}(\alpha)-m \psi_{n}^{\prime}(\alpha) \psi_{n}(m \alpha)}{\zeta_{n}(\alpha) \psi_{n}^{\prime}(m \alpha)-m \psi_{n}(\alpha) \zeta_{n}^{\prime}(m \alpha)} \\
& b_{n}=\frac{m \psi_{n}^{\prime}(m \alpha) \psi_{n}(m \alpha)-\psi_{n}(m \alpha) \psi_{n}^{\prime}(\alpha)}{m \psi_{n}^{\prime}(\alpha) \zeta_{n}(m \alpha)-\zeta_{n}^{\prime}(\alpha) \psi_{n}(m \alpha)} \\
& \psi(\xi)=\left(\frac{\pi \xi}{2}\right)^{1 / 2} J_{n+1 / 2}(\xi) \\
& \psi_{n}^{\prime}(\xi)=\frac{d \Psi n(\xi)}{d \xi} \\
& \zeta_{n}(\xi)=\left(\frac{\pi \xi}{z}\right)^{1 / 2} H^{(2)}{ }_{n+1 / 2}^{(\xi)} \\
& \zeta_{n}^{\prime}(\xi)=\frac{d \xi_{n}(\xi)}{d \xi}
\end{aligned}
$$

$\xi$ represent variables, $J_{n+1 / 2}(\xi)$ is first kind of Bessel function of half integer class, $H_{n+1 / 2}^{(2)}(\xi)$ is second kind of Hankel function of half integer class.

In which

$$
\begin{aligned}
& \psi_{-1}(\xi)=\cos \xi \\
& \psi_{0}(\xi)=\sin \xi \\
& \zeta_{-1}(\xi)=\cos \xi-i \sin \xi \\
& \zeta_{0}(\xi)=\sin \xi+i \cos \xi
\end{aligned}
$$

$\xi$ can be $k$ or $\beta$, $k$ is scale coefficients of Mie scattering simplification.

$$
k=2 \pi r / \lambda_{1}, \beta=m k
$$

$r$ is an effective radius of scattering ball here; $\lambda_{1}$ wavelength of the incident wave, $\lambda_{1}=\lambda_{0} / n_{1}, \lambda_{0}$ is the wavelength of incident light in vacuum; $n_{1}$ is refractive index of medium around scattered ball.

According to proportion relationship of radius size and wavelength of scattering particle ( $k=2 \pi r / \lambda_{1}$ ), when $k \geq 50$ that is Debyd scattering, when $k \geq 0.1$ that is Mie scattering, when $k<0.1$ that is Rayleigh scattering. In this paper, the particle we studied is mainly particles in ordinary atmosphere environment, the higher percentage dust, industrial emissions are big particle in these particles, 
combined scale coefficients are mostly in Mie scattering range. So this article focuses on Mie scattering effects on spectrum linearity.

\subsection{Planck's blackbody radiation law}

Planck's blackbody radiation law is given formula by German physicist Mr Planck in 1901 [5].

$$
M=\frac{c_{1}}{\lambda^{5}} \cdot \frac{1}{e^{\frac{c_{2}}{\lambda T}}-1}
$$

Among them, the M-blackbody spectrum radiate exitance unit $\mathrm{W} / \mathrm{m}^{2} ; \lambda$-wavelength unit $\mu \mathrm{m}$; Tthermodynamic temperature unit K; K- Boltzmann constant; H- Planck's constant $h=6.63 \times 10^{-34} \mathrm{JS}$; $c_{1}$ - the first radiation constants $c_{1}=2 \pi h c^{2}=3.749177 \times 10^{-16} \mathrm{~W} \cdot \mathrm{m}^{2}$; - the second radiation constants $c_{2}=\frac{h c}{k}=1.141235 \times 10^{-2} \mathrm{~m} \cdot \mathrm{k}$.

According to research purpose of this paper, according to the formula (13) type using the MATLAB, it is concluded that the curve distribution diagram between wavelength and radiation exitance.

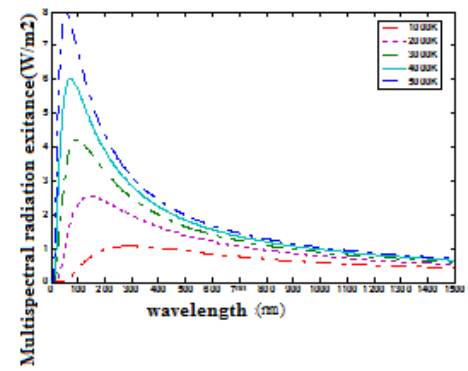

Fig.1 $1000 \mathrm{k}$ to $5000 \mathrm{k}$ blackbody multispectral radiation exitance curve

From Fig.1, we can get radiation exitance $M$ change along with the change of wavelength and continue to change, at the same time, increased with the temperature $\mathrm{T}$, proportion of short wave in blackbody radiation is increased, the proportion of long wave radiation is reduced, and move to the short-wave direction with increase of temperature $\mathrm{T}$ [6].

\section{The experimental research}

\subsection{Specific steps}

The experiment measures radiation linearity of some substance and blackbody radiation linearity fitted to get the measured temperature; Analyzed scattering medium size that influenced temperature of the material under test; attenuation spectrum of scattering medium are obtained by simulation; Uuing the measured spectrum divided by the attenuation spectrum, then get corrected after radiation spectrum; and fit again blackbody radiation linearity to get the revised temperature.

\subsection{Analysis measured spectral linearity}

Solar spectrum measurement selects the clear weather; visibility is $5 \mathrm{~km}-10 \mathrm{~km}$.

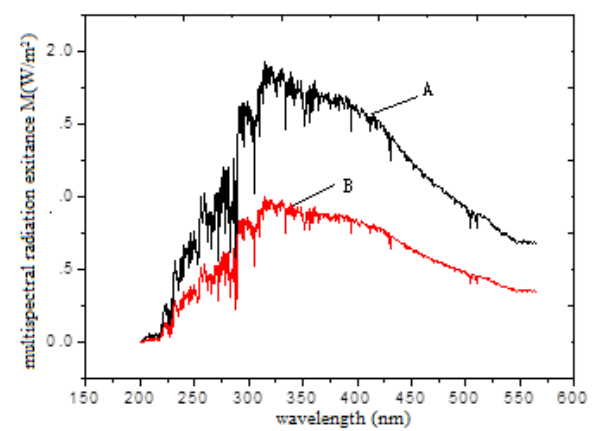

Fig. 2 Measured spectral line type of the sun

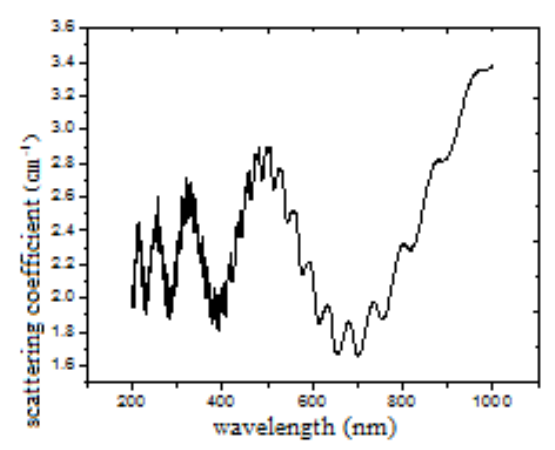

Fig.3 Relationship between PM2.5 scattering coefficient and wavelength 
(curve A is measured multispectral radiation exitance of the sun, curve B is normalization processing)

It can be seen in Fig. 2 that spectrum curve of the sun is more stable, which is maximum in the short wavelength, it shows that the temperature of the sun is mainly reflected in ultraviolet band. According to the Planck's formula, after the measured spectral line type of the sun has been done normalization processing and compare blackbody radiation temperature, found that the measured temperature of the sun at about $4600 \mathrm{k}$. But according to the data, it shows the sun's temperature is $5780 \mathrm{k}$, there are 1180 $\mathrm{k}$ deviation between the two.

The substances that influencing the sun are mainly atmospheric particles, dust, water, etc., where the impact of atmospheric particles is the key, the main reason is that the atmospheric particles absorb some light of the sun and reflect light around at the same time, they likes thousands of point light source, the sun through the reflex of the dust, strength greatly weakened, thus become soft.

Atmospheric particle according to type can be divided into fog, smoke and dust, etc, they are shown in Table 1 [7].

Table 1 The atmospheric main component content and effect

\begin{tabular}{|c|c|c|}
\hline Main components & Atmospheric content & Effect \\
\hline $\begin{array}{c}\text { Particle matter, including dust, } \\
\text { industrial emissions, etc }\end{array}$ & Unstable & $\begin{array}{c}\text { Effect on temperature and cloud } \\
\text { formation }\end{array}$ \\
\hline $\begin{array}{c}\text { Drops of water, including droplets, } \\
\text { cloud droplets, water vapor }\end{array}$ & Unstable & $\begin{array}{c}\text { Effect on formation of weather } \\
\text { conditions }\end{array}$ \\
\hline Ozone & $0.000004 \%$ & Resist the ultraviolet radiation \\
\hline Carbon dioxide & $0.037 \%$ & Keep temperature \\
\hline Oxygen & $21.95 \%$ & Life support and help combustion \\
\hline Helium & $78.08 \%$ & Plants growing necessities \\
\hline
\end{tabular}

Fog occupies about $10 \%$ of the atmosphere whose diameter is about $0.1 \mu \mathrm{m}$ to $1 \mu \mathrm{m}$, which does not meet the Mie scattering discriminant criteria. Smoke: accounts for about $20 \%$ of the atmospheric dust, under 0.5 microns in diameter, and does not meet the Mie scattering discrimination criteria. Dust: its particle diameter size is largely different, usually within $1 \mu \mathrm{m}$ m to $10 \mu \mathrm{m}$; this particle occupies $60 \%$ proportion in the atmospheric dust, which meets the Mie scattering theory and has the biggest influence on the temperature of the sun at the same time. Considering the PM2.5 index that current state repeatedly spoke, this paper selects the particle diameter with $2.5 \mu \mathrm{m}$ to analyze, and modify the actual spectral line of the sun.

It can be seen from the Fig.3, the relationship between scattering coefficient of PM2.5 particles and the wavelength is great, five change sections occur, but it is smaller in the long wavelength range, it is mainly in the short wavelength range, especially in $480 \mathrm{~nm}$ wave crest occur and have the greatest influence, the sun's energy is mainly manifested in the range of shortwave, it also proved that PM2.5 particles had a great influence on the sun's radiation temperature [8].

\subsection{Modification of spectral line}

Using the relationship between emissivity and radiation sources to modify spectrum, expression is as follows:

$$
\varepsilon_{\lambda T}=\frac{M_{\lambda T \text { 实际 }}}{M_{\lambda T \text { 黑体 }}} \text { get } M_{\lambda T \text { 黑体 }}=\frac{M_{\lambda T \text { 实际 }}}{\varepsilon_{\lambda T}}
$$

Particle impact on spectral linearity was mainly used Origin8 software to modify the results, as shown in Fig.4, where curve A the actual spectrum of the sun, curve B is the revised spectral line type.

It can be seen in the Fig.4, the change of the two curve is very similar that are at the wavelength of 300nm-350nm, radiation exitance is maximum, and multispectral radiation exitance changes largely in short wave, the modified relative deviation between the two is $10 \%$, after revise the temperature of the sun is $5100 \mathrm{k}$, it shows that by using this method to modify, the result is more accurate.

This experiment verifies when the sunlight go through atmosphere, the temperature will be affected by the PM2.5 particles in the air. At the same time, the method of temperature modification is given; it can provide some basis for measurement of the temperature of the sun. 


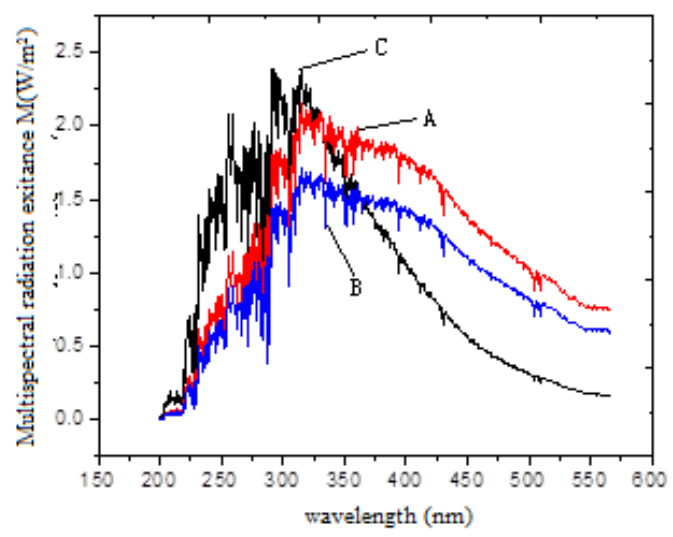

Fig.4 Revised spectral line

(curve $\mathrm{A}$ is actual radiation exitance of the sun, curve $\mathrm{B}$ is the measured radiation exitance, curve $\mathrm{C}$ is revised radiation exitance)

\section{Conclusion}

This article by measuring the solar spectrum, the temperature of the sun is measured $4600 \mathrm{k}$, but it is different from actual temperature $5780 \mathrm{k}$ of sun, what affect the temperature change of the sun are mainly dust particles in the atmosphere, this paper adopts discussion focus PM2.5 particles as the analysis object, using the scattering characteristics of PM2.5 particles modify the spectrum of the sun, the revised temperature is $5100 \mathrm{k}$ and better realize the temperature modification, feasibility of this method is verified. This method better realize the modification of spectral measuring temperature, this method can be applied to non-contact temperature measurement such as the laser explosion, burning, high temperature measurement and so on.

\section{References}

[1] Xiaogang sun, Chengwei Li, Jingmin Dai, Multispectral radiation thermometry theory review [J]. Acta Metrologica Sinica, 2005, (01).

[2] Wenge Wang. Radiation thermometry technology summary[J]. Journal of Astronautic Metrology and Measurement, 2005, (04).

[3] Hongbo Xiao, Gang Chen. Experimental research of light scattering method measuring the particle size, concentration[J]. Journal of applied optics, 2009, (04).

[4] Jinji Ma, Jin Chen. Mie scattering theory is used to calculate the atmospheric aerosol optical properties [J]. Journal of atomic and molecular physics, 2005, (04)

[5] Mingmei Wang. Dimensional analysis of blackbody radiation formula[J]. College physics, 2009, (02).

[6] Daisheng $\mathrm{Xu}$, Jiayou Tao, Song Chen. Blackbody radiation characteristics analysis based on the MATLAB [J]. Journal of Hunan Institute of Science and Technology(Natural Sciences), 2008, (04).

[7] Yili Tian, Lili Xie, Ruyu Xu. Research on dust concentration measurement [J]. Journal of chongqing university, 2003, (6): p.30-31.

[8] Ming Yang, Baorong Wang, Yingjie Li. Polarization characteristics analysis of atmospheric scattering light [J]. Journal of optoelectric technology, 2009, (02). 